\title{
Les réserves de biosphère : des lieux de collaboration entre chercheurs et gestionnaires en faveur de la biodiversité
}

\author{
Catherine Cibien \\ Secrétaire scientifique du comité MAB France, MAB France, BP 42234, 31321 Castanet Tolosan cedex, France
}

La conférence internationale «Biodiversité, science et gouvernance », organisée par la France, a sensibilisé le grand public à l'érosion rapide de la biodiversité, aujourd'hui assimilée à une sixième grande crise d'extinction (Teyssèdre, 2004). Le lien indispensable entre conservation et développement y a été réaffirmé, de même que l'importance d'approches scientifiques intégrées. La nécessité de considérer la biodiversité dans l'ensemble des activités humaines et de ne pas limiter sa conservation aux espaces protégées y est apparue évidente.

En France, une «stratégie nationale pour la biodiversité » impliquant plusieurs ministères se met en place. En conformité avec les engagements internationaux pris, elle affiche pour objectif rien de moins que l'arrêt de l'érosion de la biodiversité dans le pays en 2010. Elle comprend une stratégie de recherche sur la biodiversité pour le développement (Institut français de la biodiversité, 2004).

C'est l'occasion de se demander comment situer le programme MAB (L'homme et la biosphère) de l'Unesco dans ce contexte, et quels place et rôle son réseau de territoires d'application, les réserves de biosphère, pourrait y prendre. Ne serait-ce pas le moment de les sortir l'un comme l'autre de la confidentialité dans laquelle ils sont maintenus depuis une trentaine d'années? Il n'est pas abusif de considérer qu'ils ont joué un rôle pionnier en ces temps où l'on parle volontiers de «zones ateliers ». Un retour en arrière s'impose donc.

\section{Évolution du concept de réserve de biosphère}

\section{Les débuts}

Il n'est pas inutile de rappeler qu'en 1968, se tenait à Paris la première conférence scientifique

Auteur correspondant : catherine.cibien@mab-france.org intergouvernementale consacrée à l'utilisation rationnelle et à la conservation des ressources de la biosphère. Et de rappeler par la même occasion que l'une des recommandations faites à l'Unesco par cette «Conférence de la biosphère » était de créer un programme international de recherche sur l'homme et la biosphère, à caractère interdisciplinaire et prenant en compte les problèmes spécifiques aux pays en développement (Unesco, 1970). Ce qui fut fait et donna naissance au programme L'homme et la biosphère (Man and biosphere, ou MAB). $C^{\prime}$ est dans ce cadre qu'ont émergé, au début des années soixante-dix, les idées qui ont été à la base du concept de « réserve de biosphère » et du « réseau mondial coordonné de parcs nationaux, réserves biologiques et autres espaces protégés » (Unesco, 2003).

La naissance de ces « réserves de biosphère » est marquée par : la volonté de disposer d'échantillons d'écosystèmes et d'espèces significatifs à l'échelle mondiale à des fins de conservation, de recherche et d'éducation ; l'intérêt de disposer de ressources logistiques fondamentales pour des travaux de recherche, sur des sites où les expériences peuvent être répétées (ibid.).

L'idée prend corps avec une vigueur remarquable : de 1976 à 1981, ce ne sont pas moins de 208 réserves de biosphère qui sont inscrites dans le réseau et celui-ci couvre alors 58 pays. Il s'agit essentiellement au départ de réserves biologiques et de sites de recherche en écologie (ibid.); un nombre non négligeable d'entre eux sont sans population, ce qui pourra sembler paradoxal quelques années plus tard pour un programme sur l'Homme et la biosphère.

Mais les conférences et les groupes d'experts se succèdent, les idées se précisent. S'affirme notamment la nécessité de mieux prendre en compte les activités humaines dans les fonctions dévolues aux réserves de biosphère. En 1984, un «Plan d'action pour les réserves de 
biosphère » (Unesco, 1984) précise la démarche. On y retrouve les axes du programme originel à travers l'accent mis sur l'extension et l'amélioration du réseau, et le développement des connaissances de base pour conserver les écosystèmes et la diversité biologique. Mais, là est le fait nouveau, les réserves y sont présentées comme des zones destinées à mettre au point et démontrer des méthodes de gestion et de mise en valeur durables. Leur utilité économique et sociale pour la population locale y est affirmée. La notion de développement durable apparaît, celle de participation locale également, bien avant la Conférence de Rio : les habitants des réserves de biosphère n'y sont pas condamnés à l'inaction, mais, au contraire, encouragés à participer à la gestion. La mise en place de mécanismes facilitant la résolution des conflits est également envisagée. La nécessité de mettre en œuvre à la fois conservation, coopération avec la population, recherche et surveillance continue se traduit par une structuration spatiale des réserves de biosphère. Elles sont alors dotées d'un zonage à trois niveaux, qui permet de décliner spatialement ces fonctions : une aire centrale, légalement protégée aux termes de la loi du pays dans lequel on se trouve, est vouée à la conservation; une zone tampon, essentiellement à vocation scientifique, l'entoure ; elles sont incluses dans une zone de coopération avec la population, dont le contour est souple. Chaque réserve peut posséder plusieurs aires centrales; on parle alors de réserve en grappe.

\section{La conception actuelle de la réserve de biosphère}

Une conférence internationale d'experts, organisée à Séville en 1995, propose une vision des réserves de biosphère pour le XXI ${ }^{\text {e }}$ siècle, valorisant les connaissances acquises et mettant l'accent sur ce qui devrait être renforcé ou amélioré. Cette conférence ne propose pas de changement de doctrine, mais fixe plus précisément les «règles du jeu ». Un premier texte, le «Cadre statutaire du Réseau mondial des réserves de biosphère » (Unesco, 1996), définit les conditions pour son fonctionnement. Il définit les critères que les réserves de biosphère doivent remplir pour être désignées et rester dans le réseau. Importante nouveauté, il prévoit la révision des sites par $l^{\prime}$ Unesco tous les 10 ans, sur présentation d'un rapport remis par le pays concerné. Il autorise leur suppression $\mathrm{du}$ réseau s'ils ne remplissent pas les critères requis. L'autre texte, intitulé "La Stratégie de Séville », énumère 90 recommandations pour un bon fonctionnement des réserves de biosphère, à mettre en œuvre aux niveaux international, national et local. Rien de nouveau sur le fond, mais la forme est plus opérationnelle. Chaque réserve doit remplir des fonctions complémentaires, formulées dans une politique ou un plan de gestion :

- conserver la biodiversité naturelle et culturelle (fonction de conservation);

- tester des approches du développement durable (fonction de développement) ;

- être des espaces de recherche, de suivi continu, d'éducation, de formation, de sensibilisation et de participation locale (fonction d'appui logistique).

Ce qu'il est important de souligner ici, c'est qu'en une trentaine d'années, les réserves de biosphère, de réserves biologiques et de zones ateliers imaginées par des scientifiques pour des recherches à long terme sur l'utilisation rationnelle des ressources naturelles sont aussi devenues des outils complexes de gestion intégrée et d'aménagement du territoire, au service des populations locales (Encadré 1). Dès 1984, elles s'inscrivaient ainsi dans le cadre des principes du développement durable et ceux de la Convention sur la diversité biologique, popularisés par la Conférence de Rio en 1992. Leurs activités sont d'ailleurs en cohérence avec l'approche par écosystème que celle-ci a retenue comme cadre principal d'action (Unesco, 2000).

\section{Les réserves de biosphère en pratique}

\section{Organisation}

Une totale liberté est laissée à chaque pays d'utiliser ses outils juridiques et institutionnels nationaux pour mettre en pratique le concept sur son territoire. Certains pays ont choisi de l'inscrire dans leur droit national. Ce n'est pas le cas en France : ici, sa mise en œuvre passe par les formules et les systèmes d'organisation nationaux considérés comme juridiquement les plus appropriés. Ainsi, le cadre statutaire du Réseau mondial des réserves de biosphère précisant que leurs aires centrales vouées à la conservation doivent être constituées aux termes de dispositions légales pour la protection à long terme des éléments à conserver, en France, elles s'appuient sur différentes figures de protection qu'offre le droit français : réserve naturelle nationale ou régionale, réserve biologique domaniale de l'Office national des forêt, zone centrale de parc national, arrêté préfectoral de protection de biotope.

Le cadre statutaire demande également que des dispositions soient prises pour intéresser et associer les pouvoirs publics, les populations locales et les intérêts privés en vue de gérer l'utilisation des ressources et les activités en zone tampon, qu'un plan de gestion soit établi pour l'ensemble du territoire et qu'une autorité soit créée pour le mettre en œuvre. En pratique, il s'agit donc d'identifier, dans l'arsenal réglementaire et administratif français, un mode d'organisation répondant à ces critères. 


\section{Encadré 1. Le Réseau mondial des réserves de biosphère}

Début 2005, le Réseau mondial des réserves de biosphère, coordonné par l’Unesco, compte 457 sites localisés dans 97 pays. Vaste espace de coopération et d'échanges, il se subdivise en réseaux régionaux dont les bases sont géographiques, sociopolitiques, linguistiques ou culturelles... Ainsi, la France participe à Euromab, qui comprend l'Europe, Israël et l'Amérique du Nord. Sont souhaitées, l'émergence d'un réseau Caraibe trilingue, à forte composante insulaire, ainsi que la revitalisation d'un réseau sur le pourtour méditerranéen. Conférences, réunions techniques, échanges, ateliers... sont organisés dans le cadre de ces réseaux. Les réseaux nationaux sont plus ou moins actifs suivant les pays ; les comités MAB nationaux qui les animent peuvent aussi susciter des coopérations bilatérales.

Les tendances observées au niveau mondial, suivant en cela l'évolution de la doctrine générale, vont dans le sens de la désignation comme réserves de biosphère de territoires généralement vastes et peuplés, outils de développement et d'aménagement du territoire. Certaines des premières réserves de biosphère ne comptaient que quelques centaines ou milliers d'hectares et pas (ou presque pas) d'habitants : la réserve de Gossenköllesee en Autriche (100 ha), les 15 réserves bulgares, toutes créées en 1977, étaient des sites de recherche et/ou de conservation : elles constitueraient maintenant des aires centrales de réserves de biosphère «nouvelle génération ».

Aujourd'hui, ce sont des entités parfois extrêmement vastes et soumises à d'importantes pressions anthropiques qui sont retenues : ainsi, la Matà atlantica au Brésil s'étend sur environ 3000 kilomètres, sur 14 États, et compte quelque 100 millions d'habitants : son objectif principal est de conserver la biodiversité de la forêt atlantique et de restaurer des corridors biologiques, en protégeant les écosystèmes forestiers subsistants et en développant des pratiques sociales d'utilisation durable des ressources. Elle concerne donc la totalité de l'entité écologique, ce qui contribue à donner une gestion cohérente et efficace à un ensemble éclaté entre différents États, administrations...

Le Maroc a établi en 1998 et 2000 deux grandes réserves de biosphère, l'Arganeraie et les oasis du Sud marocain, respectivement de plus de 2,5 et 7 millions d'hectares, où le développement économique et social des populations est une question cruciale.

Aux États-Unis, la réserve de biosphère des Appalaches du Sud est un outil de promotion pour la recherche, pour la prise en considération de l'environnement et pour la valorisation des projets locaux concernant une région s'étendant sur 6 États. Conception bien différente de la France, elle est administrée par une association de droit privé qui comprend les universités, les communautés locales, des ONG.

Des territoires plus restreints qui représentent une unité géographique, écologique ou culturelle, obtiennent toutefois encore la désignation pour développer des projets de développement basés sur la valorisation des ressources locales; par exemple, deux réserves ont été reconnues récemment dans les Alpes : Grosse Wassertal en Autriche (2000) et Entelbuch en Suisse (2001), respectivement sur 20000 et 40000 hectares environ. En Espagne, plusieurs îles (Lanzarote et El Hierro aux Canaries, Minorque aux Baléares) ont été désignées en totalité « réserves de biosphère ».

C. Gruau (2002) considère que, contrairement à ce qu'il en était pour celui des parcs nationaux mis en place en application de la loi de 1960, le statut de parc naturel régional répond bien à cette exigence. On doit toutefois noter que le rapport du député J.-P. Giran sur les parcs nationaux (Giran, 2003), et le projet de loi qui en découle, préfigurent des parcs nationaux «nouvelle génération » plus proches $\mathrm{du}$ concept de réserve de biosphère. En effet, si l'on suit ces textes, des moyens plus importants devraient être accordés à la zone périphérique et une véritable politique contractuelle favorisant la participation locale et le développement durable autour du «cœur du parc » (proche du concept d'aire centrale) devrait être mise en place. Ces évolutions convergentes sont très significatives d'un mouvement d'ensemble allant vers une nouvelle articulation de la protection et du développement.

Dans certains cas, des blocages politiques, des frilosités, des conflits d'intérêts... empêchent la mise en œuvre des structures les plus habituelles pour s'engager dans des projets de développement territorial. Les espaces protégés, même de façon contractuelle, font quelquefois l'objet de rejets de la part des acteurs locaux qui craignent des restrictions. Quand il s'agit d'associer des acteurs variés à un même projet, des formules souples (type association, fondation, consortium...) sont alors explorées. Se pose toutefois la question de leur visibilité, ainsi que celle de leur capacité à mobiliser des moyens dans la durée. Dans certains cas, les pouvoirs politiques portent directement le projet de la réserve de biosphère, comme par exemple sur l'île de Minorque (Espagne).

Pour ce qui concerne la France, l'intérêt du concept de réserve de biosphère est qu'il permet d'intégrer, dans des dosages variables correspondant à la diversité des situations, différents dispositifs de protection et de gestion de la biodiversité, et des outils de développement. Il permet de donner de la cohérence à un système de gestion complexe, qui reflète bien la complexité des enjeux de tout territoire. Par contre, il entraîne inévitablement une superposition de labels (par exemple, un parc naturel régional reconnu en plus comme réserve de biosphère); et ceci peut être troublant et gênant. En effet, si ces appellations multiples ont un sens clair pour les spécialistes, elles entraînent une perte de lisibilité aux yeux du plus grand nombre. Et ceci importe tout particulièrement s'agissant des autorités et de la population locales qui sont supposées être impliquées dans l'action de protection portant sur l'espace concerné. Il leur est parfois difficile de comprendre l'intérêt de cette double appellation. 


\section{Encadré 2. Le Réseau national des réserves de biosphère}

Le réseau des réserves de biosphère françaises compte dix territoires (Tab. 1). Progressivement établi depuis 1977, il révèle l'évolution du concept. Les premiers sites désignés étaient la Camargue, réserve nationale strictement protégée, sans habitants, vouée à la conservation, et l'atoll de Taiaro dont les caractéristiques morphologiques lui confèrent un intérêt particulier en matière de recherche, notamment en biologie des populations marines. Aujourd'hui, ces deux réserves de biosphère sont en cours de totale refonte, car elles ne répondent pas au concept dans sa forme actuelle et devraient en être exclues, faute d'évolution. Les territoires désignés plus récemment donnent la priorité à un développement durable respectueux des patrimoines naturels et culturels, parfois soumis à de fortes pressions d'urbanisation (le Luberon ou Fontainebleau, par exemple).

Les réserves de biosphère françaises sont liées au système des espaces protégés du ministère en charge de l'environnement : cinq d'entre elles sont portées d'un point de vue administratif et financier, entièrement ou en partie, par des parcs naturels régionaux et deux par des parcs nationaux. Les réserves du mont Ventoux et du Pays de Fontainebleau échappent à cette « règle ". La seule désignation de « réserve de biosphère » pour le mont Ventoux ne lui a pas permis jusqu'à présent d'accéder à des soutiens publics suffisants pour mettre en œuvre l'ensemble de ses objectifs. Un parc naturel régional est en cours de préfiguration. À Fontainebleau, une association loi 1901 vient d'être constituée comme structure support de la réserve de biosphère. On peut se demander si associer principalement les réserves de biosphère au système des espaces protégés du ministère de l'Écologie n'obère pas leur fonction de développement économique et social, les «cantonnant » à être des espaces protégés alors que la Stratégie de Séville stipule qu'elles sont «plus que des espaces protégés ».

La réserve de biosphère des Vosges du Nord est intégrée depuis 1998 dans la réserve de biosphère transfrontalière Vosges du Nord - Pfälzerwald. Ce type de coopération ouvre d'intéressantes perspectives sur les plans économique, social, culturel et écologique, et représente un enjeu important, particulièrement dans le contexte de la construction européenne : la réflexion sur les aspects institutionnels des réserves de biosphère, par exemple, est récente; elle demande à être développée. Cette question n'intéresse pas seulement les territoires transfrontaliers, elle concerne aussi ceux qui aspirent à devenir réserve de biosphère et pour lesquels un statut et une organisation adéquats doivent être trouvés : le parc naturel régional serait-il la seule réponse à la française (sous réserve de ce que seront les futurs parcs nationaux) ou des systèmes plus souples, comme il s'en rencontre à l'étranger (consortium, association d'acteurs publics et privés), ont-ils un rôle moteur à jouer pour porter un projet territorial de conservation et de développement durable?

La Stratégie de Séville recommande aux pays de procéder à une analyse biogéographique comme base d'un réseau représentatif. Une telle étude n'a pas été menée en France. Le comité français du MAB ne suscite généralement pas de projets de réserves de biosphère en fonction de considérations biogéographiques, mais privilégie l'accompagnement de ceux qui émanent du « terrain » et présentent des qualités en termes de patrimoines naturel et culturel, ainsi que de projet.

Il est donc nécessaire d'améliorer la visibilité des réserves de biosphère par rapport aux structures nationales et de leur donner la place légitime qui leur manque aujourd'hui (Encadré 2). En période de développement rapide du processus de mondialisation, et compte tenu, dans ce contexte, de la difficulté de mise en œuvre du développement durable, $c^{\prime}$ est une chance de disposer d'un tel outil d'expérimentation au niveau local. Il est précieux de pouvoir bénéficier de la longue et riche réflexion qui a abouti à la conception que l'on en a aujourd'hui, du caractère très élaboré du dispositif de recherche et d'action qu'il constitue, de l'expérience qu'il a acquise tant sur le plan scientifique que pratique, de sa grande souplesse qui permet de l'adapter aux contextes les plus variés et, enfin, de sa portée et de sa reconnaissance internationales. Les réserves de biosphère intègrent les éléments reconnus comme primordiaux pour limiter l'érosion de la biodiversité : une approche basée sur la gouvernance locale, un fonctionnement international en réseaux, une grande place laissée à la créativité quant à l'organisation. . .

\section{Place de la recherche dans les réserves de biosphère}

En trente années d'existence, la place de la recherche dans les réserves de biosphère a beaucoup évolué. Les premières d'entre elles étaient des réserves biologiques pures et simples, quand elles n'étaient pas carrément des stations de relevés et d'expérimentation de laboratoires de recherche : elles privilégiaient donc deux fonctions, celle de conservation et celle de recherche scientifique. Aujourd'hui, suivant en cela la doctrine de la Stratégie de Séville, elles en affichent trois : conservation, développement et logistique. On peut dire des réserves de la biosphère françaises qu'elles prennent bien en charge les deux premières; en effet, proches du monde des gestionnaires d'espaces naturels, elles portent des projets territoriaux de conservation et de développement. On constate, par contre, que la fonction logistique pourrait y être améliorée ; et ceci vaut tout particulièrement pour tout ce qui touche à la recherche et au suivi scientifiques. En effet, les textes-cadres proposent d'utiliser le réseau des réserves de biosphère pour des programmes nationaux et internationaux de recherche traitant de la diversité biologique, des connaissances locales en la matière, de la désertification, du cycle de l'eau, des changements globaux. Ils encouragent le développement d'outils de recherche interdisciplinaires et novateurs, y compris les modèles d'intégration de données sociales, écologiques et économiques. Il recommande au niveau national d'intégrer les réserves de biosphère dans les programmes de recherche nationaux et régionaux. 
Tableau 1. Les réserves de biosphère françaises.

\begin{tabular}{|c|c|c|c|}
\hline $\begin{array}{l}\text { Nom de la réserve de } \\
\text { biosphère }\end{array}$ & $\begin{array}{l}\text { Date de } \\
\text { création }\end{array}$ & $\begin{array}{c}\text { Structure } \\
\text { coordinatrice }\end{array}$ & Remarques \\
\hline Atoll de Taiaro & 1977 & À établir & $\begin{array}{l}\text { En révision, en projet la réserve de biosphère des } \\
\text { Tuamotu comprenant } 7 \text { atolls, dont Taiaro, et } \\
6 \text { atolls habités }\end{array}$ \\
\hline Camargue & 1977 & $\begin{array}{l}\text { À établir, suivi } \\
\text { de la révision } \\
\text { assuré par le Parc } \\
\text { naturel régional } \\
\text { de Camargue }\end{array}$ & $\begin{array}{l}\text { En révision complète, extension si possible à } \\
\text { l'ensemble du delta du Rhône. Suppression si pas } \\
\text { d'accord local }\end{array}$ \\
\hline Vallée du Fango & 1977 & $\begin{array}{l}\text { Parc naturel } \\
\text { régional de Corse }\end{array}$ & Révisée et agrandie en 1990 \\
\hline Cévennes & 1984 & $\begin{array}{l}\text { Parc national des } \\
\text { Cévennes }\end{array}$ & \\
\hline Mer d'Iroise & 1988 & $\begin{array}{l}\text { Parc naturel } \\
\text { régional } \\
\text { d'Armorique }\end{array}$ & Élargissement à l'île de Sein à l'étude \\
\hline Vosges du Nord & 1988 & $\begin{array}{l}\text { Parc naturel } \\
\text { régional des } \\
\text { Vosges du Nord }\end{array}$ & $\begin{array}{l}\text { Intégrée à la réserve de biosphère transfrontalière } \\
\text { Vosges du Nord - Pfälzerwald en } 1998\end{array}$ \\
\hline Mont Ventoux & 1990 & $\begin{array}{l}\text { Syndicat mixte } \\
\text { d'aménagement } \\
\text { et d'équipement } \\
\text { du mont Ventoux }\end{array}$ & \\
\hline Archipel de Guadeloupe & 1992 & $\begin{array}{l}\text { Parc national de } \\
\text { Guadeloupe }\end{array}$ & \\
\hline Luberon & 1997 & $\begin{array}{l}\text { Parc naturel } \\
\text { régional du } \\
\text { Luberon }\end{array}$ & \\
\hline Pays de Fontainebleau & 1998 & $\begin{array}{l}\text { Association de } \\
\text { coordination de } \\
\text { la réserve de bio- } \\
\text { sphère du Pays } \\
\text { de Fontainebleau } \\
\text { et du Gâtinais } \\
\text { français }\end{array}$ & \\
\hline
\end{tabular}

Alors qu'elles ont été créées par des chercheurs, les réserves de biosphère sont aujourd'hui un peu désertées par eux. Au niveau national, il est très significatif de constater que la mise en place des zones ateliers du CNRS a été conduite sans la moindre référence aux réserves de biosphère : elles ne suscitent donc guère l'intérêt ; inversement, il n'y a pas eu de demande de leur part à cette occasion : une seule exception est à noter, celle de la réserve de biosphère des Cévennes (mais, en réalité, c'est au titre du Parc national que la démarche a été faite). Pourtant, la convergence entre les concepts de base des réserves de biosphère et ceux des zones ateliers est importante si on s'en tient à la définition de ces dernières : réseaux coordonnés à différents niveaux de zones définies à l'échelle régionale, ayant une certaine unité fonctionnelle, privilégiant des démarches interdisciplinaires, pouvant répondre à des questionnements scientifiques issus d'une demande sociale ${ }^{1}$. Ceci montre bien que les réserves de biosphère ne sont pas, aujourd'hui, considérées par la

1 http://www.cindy.ensmp.fr/PEVS2/za01.htm communauté scientifique comme les territoires privilégiés de recherche qu'elles sont supposées être.

Réciproquement, les parcs, structures support de la plupart des réserves de biosphère, $n$ 'intègrent pas la recherche comme une de leurs fonctions prioritaires, au même titre que conservation et développement. Ils ne sont pas suffisamment équipés pour pouvoir remplir eux-mêmes cette fonction telle qu'elle est prévue pour les réserves de biosphère. Les parcs nationaux ont des services et des conseils scientifiques, mais n'ont pas de mission de recherche proprement dite. Ce n'est que récemment que les parcs naturels régionaux français ont eu l'obligation de se doter de conseils scientifiques, et tous n'ont pas de personne formée à la recherche dans leur personnel, sans même parler d'un service scientifique.

Les territoires de certaines réserves de biosphère françaises font depuis longtemps l'objet d'investigations de la part de nombreuses équipes scientifiques (Camargue, Cévennes, Fango, Mont Ventoux, par exemple), mais les liens avec les structures gestionnaires et un relais à ces travaux ne sont pas toujours suffisants. Certaines ne 
peuvent pas fournir une liste actualisée des travaux de recherche effectués sur leur territoire. Elles n'ont pas de stratégie ou de programme de recherche proprement dit, structuré, formulé, adapté à leurs besoins. Les actions de gestion ne s'appuient pas toujours sur des résultats scientifiques. Quand l'appui scientifique existe, il est le produit de relations avec des équipes présentes sur le territoire de la réserve ou avec des réseaux des chercheurs impliqués dans les comités scientifiques. Parfois, des travaux de recherche sont initiés ou orientés par la réserve de biosphère, grâce à des financements le plus souvent ponctuels. La relation entre l'équipe de recherche et la structure de gestion du territoire n'est pas toujours optimale ; elle est même parfois quasiment inexistante, et souvent objet de frustrations réciproques ou de malentendus. En effet, les gestionnaires attendent des chercheurs des réponses claires, précises, à brève échéance, aux questions (quelquefois mal formulées) qu'ils se posent, alors que le travail des chercheurs développe des questionnements. Les gestionnaires ont souvent des demandes qui relèvent plus de l'expertise ou de l'étude que de la recherche. L'ambiguité sur les fonctions de chacune de ces formes de rapport entre chercheurs et gestionnaires demande à être levée.

Parallèlement, les chercheurs reprochent aux gestionnaires leur manque de disponibilité et d'appui logistique. Le gestionnaire doit faire face à de nombreuses urgences ; il est soumis à des contraintes de temps; il a une obligation de résultats rapides : autant de conditions de travail auxquelles le chercheur est moins directement confronté. L'investissement dans la durée et dans la régularité est souvent difficile pour un gestionnaire, dont l'activité repose sur la gestion simultanée de nombreux dossiers. Ajoutons pour finir que tous ne sont pas en mesure d'offrir un appui logistique (logement, matériel, aide sur le terrain) ou des financements significatifs ou pérennes.

Ceci ne doit pas faire oublier que les réserves de biosphère offrent à une équipe de recherche des conditions de travail présentant un intérêt certain par rapport à celles d'un territoire "ordinaire». En premier lieu, elles sont dotées de politiques de gestion claires et formulées, intégrant conservation et développement ; elles sont donc un terrain privilégié pour des recherches autour de cette articulation. Par ailleurs, elles disposent souvent de données relatives à des domaines variés que l'on ne trouve pas partout (inventaires naturalistes, paysagers, culturels, données socioéconomiques... ). Et elles sont dotées d'une équipe technique formée, en contact avec les scientifiques du conseil. Enfin, elles sont un lieu d'interface avec la population, ses élus, ses représentants associatifs.

Une véritable intégration de la fonction de recherche dans les réserves de biosphère permettrait des gains réciproques sur deux plans:

- Sur le plan local tout d'abord. La gestion de territoires dans une perspective de développement durable requiert d'importantes capacités d'analyse, puisqu'elle nécessite d'adapter (parfois de modifier totalement) des politiques, des pratiques, des incitations, etc., que ce soit en matière de gestion d'espaces et d'espèces ou de développement. Anticiper, accompagner, orienter plutôt que subir les mutations à l'œuvre, tel est le cœur du travail du gestionnaire. Il est donc essentiel pour lui de saisir les dynamiques en cours sur son territoire, et celles que celui-ci subit de ce qui l'entoure (au sens large). Par dynamiques, on entend les dynamiques écologique, sociale, économique et culturelle, ainsi que leurs interactions. Ce cadre large implique des travaux inscrits dans la durée, concernant des thèmes très variés, le plus souvent pluridisciplinaires.

La formulation de programmes de recherche pertinents pour la gestion nécessite une interaction forte des deux parties (gestionnaires et chercheurs), car elle suppose d'importantes clarifications d'objectifs et de langage. Une condition sans doute indispensable pour faire que ce dialogue se déroule à la satisfaction de tous est qu'il se fasse à travers une forme de cohabitation durable et active. C'est dans l'esprit de favoriser une meilleure collaboration entre recherche et gestion que le comité MAB France a engagé un projet financé par le ministère de l'Écologie et du Développement durable. Ce projet comprend deux volets. Le premier vise à une sensibilisation réciproque des gestionnaires et des chercheurs : les premiers, aux différentes disciplines des sciences de la conservation; les seconds, aux contraintes des gestionnaires (Schmitzberger et al., à paraître). Le deuxième volet consiste en une expérience originale de construction d'un programme-cadre de recherche dans une réserve de biosphère (en l'occurrence celle des Vosges du Nord). Cette expérience présente une double originalité : celle de faire intervenir un médiateur entre le gestionnaire et le comité scientifique dans le processus de construction du programme; celle de se référer explicitement et simultanément à la Stratégie nationale de recherche sur la biodiversité pour le développement durable, à la Stratégie de Séville et à la charte du Parc naturel régional, document de cadrage décennal de la gestion du territoire.

Signalons que, par ailleurs, l'Institut français de la biodiversité finance un projet de recherche reposant sur la démarche dite de "modélisation d'accompagnement multi-agents », en vue de l'aide à la gestion, dans trois réserves de biosphère (M. Étienne, coordinateur). À travers cette recherche, les personnes impliquées, scientifiques comme gestionnaires, sont conduites, via la modélisation, à élaborer des visions partagées des problèmes et à aller dans le sens de choix de gestion concertés en travaillant sur des scénarios conçus en commun.

- Aux niveaux national et international ensuite. Si l'on veut qu'en matière de biodiversité, la prise de décision politique, que ce soit au niveau national ou international, repose sur des données scientifiques, un observatoire 
national doit être mis en place pour pouvoir procéder à une évaluation périodique de son état et pour assurer le suivi de ses évolutions en utilisant des indicateurs validés (Couvet et al., 2004). De ce point de vue, le dispositif des réserves de biosphère a un rôle évident à jouer, en participant d'ores et déjà à la collecte de données, même si, dans certaines réserves, un appui est nécessaire pour l'améliorer.

\section{Conclusion}

Les réponses à apporter aux questions posées par le souci de concilier la préservation de la biodiversité et les exigences du développement appellent un effort particulier et important de recherche. Dans ce contexte, le programme MAB et son réseau de réserves de biosphère montrent toute la pertinence de la démarche qu'ils proposent depuis trente années, tant sur le plan conceptuel que sur le plan pratique. Parties prenantes du seul réseau mondial de territoires constitué d'unités d'aménagement et de développement structurées, intégrant la notion de corridor biologique, s'appuyant sur des démarches locales en réseau, elles constituent un outil mature au service de la conservation de la diversité biologique et du développement durable.

Le fait que la mise en pratique du concept de réserve de la biosphère ait pu être parfois décevante doit-il conduire à son abandon? Ces déconvenues témoignent certes de la difficulté de sa mise en œuvre (difficulté également observée pour l'orientation de nos sociétés vers plus de durabilité), mais elles témoignent aussi de la faiblesse du soutien que les réserves de biosphère existantes ont reçu en tant que telles des pouvoirs publics; ceci vaut notamment pour la France, que ce soit au niveau local ou au niveau de la coordination nationale. La souplesse de leur statut est une faiblesse face aux réalités administratives françaises. Elles sont plus considérées comme un label honorifique que comme un outil de gestion adaptatif et innovant, et les moyens financiers dont elles disposent sont en conséquence.

En toute logique, l'application de la Stratégie nationale pour la biodiversité devrait faire qu'il leur soit accordé une place et un rôle plus importants dans la recherche et dans l'action. Il faut espérer qu'il en sera ainsi afin qu'elles puissent devenir effectivement les lieux d'une « recherche engagée » (Barbault, 2003) en faveur de la conservation de la biodiversité et du développement durable qu'elle sont censées être.

\section{Remerciements}

Remerciements à Robert Barbault pour sa lecture dynamisante et ses conseils.

\section{Références}

Barbault, R., 2003. La biologie de la conservation dans les réserves de biosphère, Lettre de la biosphère, 66, 1-2.

Couvet, D., Jiguet, F., Julliard, R., Levrel, H., 2004. Les indicateurs de biodiversité, in Barbault, R., Chevassus-au-Louis, B. (Eds), Biodiversité et changements globaux : enjeux de sociétés et défis pour la recherche, Paris, ADPF, ministère des Affaires étrangères, 40-46.

Giran, J.-P., 2003. Les Parcs nationaux, une référence pour la France, une chance pour les territoires. Rapport au Premier ministre.

Gruau, C., 2002. La Gestion durable des réserves de biosphère françaises. Considérations sur les outils juridiques pertinents pour une gestion des réserves de biosphère sur le territoire français. DEA en droit de l'environnement, Université de Nantes.

Institut français de la biodiversité, 2004. Stratégie nationale de recherche sur la biodiversité au service du développement durable, Paris, IFB.

Schmitzberger, K., Winterton, M., Cibien, C., à paraître. Les sciences de la conservation, appui à la gestion des territoires.

Teyssèdre, A. 2004. Vers une sixième grande crise d'extinctions?, in Barbault, R., Chevassus-au-Louis, B. (Eds), Biodiversité et changements globaux : enjeux de sociétés et défis pour la recherche, Paris, ADPF, ministère des Affaires étrangères, 24-36.

Unesco, 1970. Utilisation et conservation de la biosphère. Actes de la Conférence intergouvernementale d'experts sur les bases scientifiques de l'utilisation rationnelle et de la conservation des ressources de la biosphère, Paris, 4-13 septembre 1968, Paris, Unesco.

Unesco, 1984. Plan d'action pour les réserves de biosphère, Nature et ressources, 20, 4, 1-12.

Unesco, 1996. Réserves de biosphère : la stratégie de Séville et le cadre statutaire du réseau mondial, Paris, Unesco.

Unesco, 2000. La Solution du puzzle : l'approche écosystémique et les réserves de biosphère, Paris, Unesco.

Unesco, 2003. Réserves de biosphère : des lieux privilégiés pour les hommes et la nature, Paris, Unesco. 